\title{
Evaluating the effects of probiotics in pediatrics with recurrent abdominal pain
}

\author{
Parisa Rahmani, $M D^{1}$, Azin Ghouran-Orimi, MD $^{2}$, Farzaneh Motamed, MD $^{1}$, Alireza Moradzadeh, MD $^{3}$ \\ ${ }^{1}$ Pediatric Gastroenterology and Hepatology Research Center, Tehran University of Medical Sciences, Tehran, Iran; ${ }^{2}$ Iran university of Medical Sciences, Tehran, Iran; \\ ${ }^{3}$ Department of Pediatric Gastroenterology, Children's Medical Center, Tehran University of Medical Sciences, Tehran, Iran
}

\begin{abstract}
Background: Recurrent abdominal pain (RAP) is one of the frequent complaints in general practice, particularly in pediatrics and is among the common cause of referral to gastroenterology clinics.

Purpose: This study is designed to investigate the effects of probiotics for the treatment of RAP and desired therapeutic outcomes.

Methods: One hundred twenty-five children with the diagnosis of RAP according to Rome III criteria for irritable bowel syndrome (IBS), functional abdominal pain (FAP), functional dyspepsia (FD), and abdominal migraine (AM), were enrolled in this double-blind randomized controlled trial.

Results: Sixty-five subjects received probiotics, and others received placebo treatment for 4 weeks. Lactobacillus renteri was therapeutically effective in 32 patients compared to 8 patients, responding to the placebo treatment. Compared to baseline, all pain-related variables showed a significant reduction for the IBS and FD at the end of the 4th week. However, it did not respond well in FAP and AM groups. Pain-related outcomes such as, frequency of the pain, severity, and duration of the pain were decreased following the probiotic treatment. No therapeutic response was seen in AM group after the administration of probiotics. L. reuteri significantly led to pain relief in the overall population, and also in FAP, FD, and IBS subgroups. Conclusion: $L$. reuteri probiotics are likely to lead to RAP relief and can be recommended for the treatment of functional gastrointestinal disorders.
\end{abstract}

Key words: Lactobacillus reuteri, Recurrent abdominal pain, Child

\section{Key message}

Question: ecurrent abdominal pain (RAP) is a chief complaint among pediatrics and is associated with reduced quality of life, for both parent and child, and economic burden. Does probiotics reduce the frequency of RAP among children?

Finding: This study reported the effects of Lactobacillus reuteri probiotics among children with RAP as a result of multiple etiologies.

Meaning: The administration of probiotic supplements is significantly associated with pain relief among RAP children presented with functional abdominal pain, irritable bowel syndrome, and functional dyspepsia.

\section{Introduction}

Gut microbiota is an important determinant of gastrointestinal health. Alterations in this diverse collection of microbes can lead to several gastric and extragastric diseases. Probiotics are living microorganisms that can be beneficial to the health of the host, if provided in an appropriate amount. ${ }^{1)}$ These microorganisms include lactic acid bacilli species (such as Lactobacillus and Bifidobacterium), nonpathogenic Escherichia coli species (e.g. E. coli strain Nissle 1917), Clostridium butyricumi, Streptococcus salivarius, and Saccharomyces boulardi (noninfectious yeast species). Genetically-engineered bacteria have immunomodulatory characteristics such as stimulating the production of interleukin-10 (IL-10) and the trefoil factor, which have beneficial effects on the immune system. ${ }^{2)}$ Several studies have shown that probiotics are effective against numerous pathological conditions. $^{3)}$

Recurrent abdominal pain (RAP) is one of the most common consequences of pediatric gastrointestinal tract infections, ${ }^{4)}$ which affects $10 \%$ of all children and the highest incidence is among 7 and 12 years old children. ${ }^{5)}$ Four to twenty percent of school-going children are presented with the complaint of RAP that prevents their daily activities ${ }^{(6)}$ such as schooling ${ }^{7)}$ perhaps due to inadequate treatment. ${ }^{8)}$ Anxiety in parents and frequent doctor referral imposes an economic and emotional burden, and results from quantitative studies have shown that children with RAP are unlikely to respond to any definitive treatment plan. Owing to the beneficial effects of probiotics on the gastrointestinal tract, immune system, the aim of this study is to investigate the effects of $L$. reuteri in the treatment of RAP in children aged 6

Corresponding author: Alireza Moradzadeh, MD. Department of Pediatric Gastroenterology, Children's Medical Center, Tehran University of Medical Sciences, Tehran, Iran 凶E-mail: md.moradzadeh.a@gmail.com, https://orcid.org/0000-0003-3355-7086

Received: 17 December, 2019, Revised: 29 April, 2020, Accepted: 20 May, 2020

This is an open-access article distributed under the terms of the Creative Commons Attribution Non-Commercial License (http://creativecommons.org/licenses/by$\mathrm{nc} / 4.0 /$ ) which permits unrestricted non-commercial use, distribution, and reproduction in any medium, provided the original work is properly cited.

Copyright (c) 2020 by The Korean Pediatric Society 


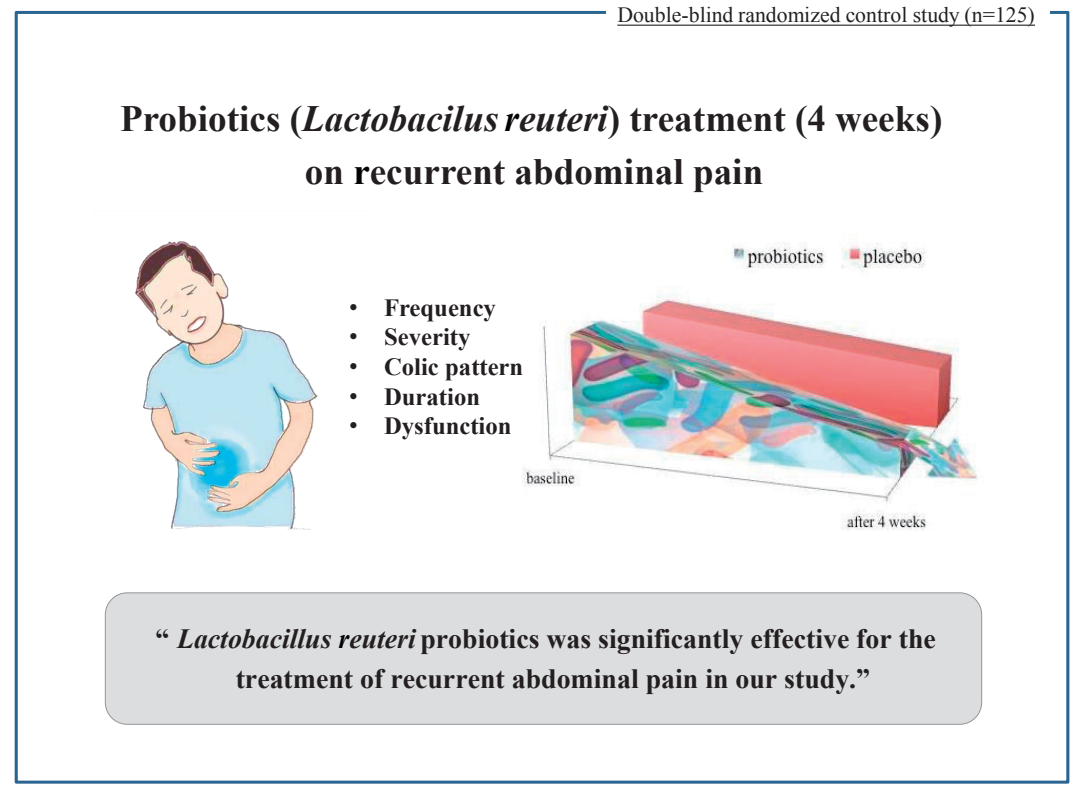

Graphical abstract

to 16 years old, referred to the gastroenterology clinic.

\section{Methods}

This is a double-blinded clinical trial that was conducted from June 2017 to June 2018. A total of 198 children with RAP signs referred to the gastroenterology clinic were randomly divided into case and control groups. Children who were included in the study were between the ages of 6 and 16 years, and the diagnosis of nonorganic RAP was performed according to ROME III criteria. $^{9)}$

Data collection method: The information collected included age, sex and weight of the child, the frequency of repetitive pain, the severity of pain, the duration of each episode of pain, the number of days drugs were administered to treat the pain, number of days of disturbance in daily activity, the type of primary disease and the pattern of pain mentioned by the doctor and recorded on a daily basis by the parents in the notebook.

The following were excluded from the study: the presence of any one of the red flag items, use of antibiotics in the last 1 month, organic disorder based on clinical and paraclinical findings, and participants or parents who did not co-operate in regards with medications and referrals.

At the time of referral, the children were physically examined and the following information was registered at the time of registration and end of the study by single physician: pain intensity based on the Wang-Baker FACES Pain Rating Scale (WBFPRS) 2 , frequency of pain and recurrence, duration of each episode of pain, pattern of pain (colic Crampi or permanent), the number of days affected by day-to-day activities (such as school absenteeism) and the need for other medications to relieve pain. The children were randomly assigned into 2 groups as quadruple blocks of case and control using Block-Randomization method. They were treated for 4 weeks with probiotic chewable tablets (containing 108 colony forming units L. reuteri) or placebo for 2 times a day. The boxes containing placebo and probiotic were similar in the shape, size, and taste.

The patients' parents were provided with the notebook to record information such as pain intensity based on WBFPRS criteria, frequency of repetitive pain per day, duration of each episode of pain, type of the pain, interruption of daily activities, and the need for the use of another drug.

Patients were examined every 2 weeks and the changes in the cases recorded by the parents were examined where, parents were questioned regarding the relief in the pain. According to the basic principles of the Helsinki Declaration, the information was recorded confidentially; complete details of the study were given to the patients and written consent was obtained for each participating individual. Patients' data were kept confidential and were not disclosed to any individual or legal person. No intervention was none to harm the patient and no additional costs were imposed on patients. This study was approved by the Research Ethics Board of Tehran University of Medical Sciences (TUMS.91/D/130/378).

The data was computerized and analyzed using IBM SPSS ver. 18.0 (IBM Co., Armonk, NY, USA). The mean of quantitative data, as the central index and standard deviation, was reported as a dispersion index. Mann-Whitney-Wilcoxon test was used for comparative analysis for the following parameters; age, weight, frequency of repetitive pain, pain intensity, duration of episode of pain, number of days drugs were used and number of days needed to use the drug and the number of days of disturbed daily activities, between the 2 groups. Chi-square or Fisher exact test was used to determine the relationship between the type of primary disease and the pattern of pain in the case and control groups. $P$ value of $<0.05$ was considered significant. 


\section{Results}

After the following the exclusion criteria and inability of patients to adhere to the study, a total of 125 subjects were studied where, 57 children had FAP, 29 had FD, 30 had IBS, and 9 were presented with AM. Overall, 65 patients with an average age of $7.3 \pm 1.7$ years in case group and 60 patients with an average age of $7.7 \pm 2.1$ years were in the control group. The mean body weight in the case group was $23.1 \pm 7.6 \mathrm{~kg}$ and in control group was $23.5 \pm 5.6 \mathrm{~kg}$. The male to female ratio was $27 / 38$ in the case group and 30/30 in the control group. Above mentioned variables (age, weight, and sex) were not significantly different between the 2 groups $(P>0.05)$. The frequency of repeated initial pain in the group receiving probiotic was $6.8 \pm 6.8$ and in the placebo (control) group was $7.8 \pm 5.9$, which was not statistically significant $(P=0.59)$. Frequency of repetitive pain 4 weeks after these 2 groups was $3.6 \pm 2.2$ and $4.6 \pm 4.9$, respectively, which was found to be statistically significant $(P<0.001)$.

In this study, success in the treatment that was defined as pain intensity $=0$ (soft-faced child or facial scan $=0$ ) was shown as the result of $L$. reuteri probiotics intake in the study population.

The severity of the primary pain prior to the treatment in the case and control groups was $3.3 \pm 0.9$ and $3.1 \pm 0.6$, respectively, which did not show a statistically significant difference between the 2 groups $(P=0.19)$. However, there was a significant difference between the severity of pain 4 weeks after the treatment in the 2 groups $(P<0.001) ; 1.3 \pm 1.1$ in the case group compared to $1 \pm 2$ in the control group.

Sixty-two patients in the case group and 58 patients in the control group, had the colic pain and 3 cases in the case group and 2 in the control group had constant pain, which was not statistically significant between the 2 groups.

Four weeks after the start of the treatment, 31 patients in the case group and 50 in the control group had colic pain, and 2 in each group had continuous pain. The pain pattern following 4 weeks was significantly different between the 2 groups $(P<$ $0.001)$.

Duration of the pain in the 2 groups was $50.8 \pm 22.5$ and $52.6 \pm 20.5 \mathrm{~min} /$ day in the case and control groups, respectively, which was not statistically significant $(P=0.25)$; while the duration of pain after 4 weeks of the treatment in these 2 groups were $15.2 \pm 5.6$ and $42.4 \pm 15.4$, respectively, which was statistically significant $(P<0.001)$.

Thirteen patients in the case group and 8 patients in the control group were reported to present disturbances in their daily activities before treatment, which was not significantly different $(P=0.34)$, whereas, 4 weeks following the treatment, 1 patient in the case group and 6 in the control group continued to have disturbed daily activities. This difference was not significant between the 2 groups $(P=0.054)$. Patients in the pretreatment group had an average daily activity of $1.2 \pm 0.5$ days per week, which was $0.7 \pm 0.2$ in the control group. The difference was not statistically significant $(P=0.25)$. Following the 4 weeks of the treatment the amount of distraction in daily activities in the case group was $0.3 \pm 0.05$ days a week and in the placebo was $0.6 \pm 0.2$ days per week, which was marked with statistical significance $(P=0.04)$.

Two patients in the case group with an average of $0.6 \pm 0.1$ days/wk required drugs for the pain management, at the beginning of the study whereas, a week after the commencement of the treatment, 1 case for $0.2 \pm 0.03$ days/wk needed drugs for pain management. There was no significant difference in the need for medication before and after treatment between the 2 groups $(P>0.05)$.

The success rate in treatment was $32(49 \%)$ in the case group and $8(13 \%)$ in the control group, which was significantly different in the 2 groups $(P<0.001)$. All data and statistical analysis are summarized in Table 1 for all participants in the study at the time of entry and after 4 weeks.

Table 1. Characteristics of all study participants by study group

\begin{tabular}{|c|c|c|c|}
\hline Characteristic & $\begin{array}{l}\text { Probiotic } \\
(n=65)\end{array}$ & $\begin{array}{c}\text { Placebo } \\
(n=60)\end{array}$ & $P$ value \\
\hline Age (yr) & $7.3 \pm 1.7$ & $7.7 \pm 2.1$ & 0.43 \\
\hline Body weight (kg) & $23.1 \pm 7.6$ & $23.5 \pm 5.6$ & 0.26 \\
\hline Sex, male:female & $27: 38$ & $30: 30$ & 0.37 \\
\hline \multicolumn{4}{|l|}{ Frequency of pain } \\
\hline At the baseline & $8.6 \pm 6.0$ & $7.8 \pm 5.9$ & 0.59 \\
\hline After 4 weeks & $2.2 \pm 3.6$ & $4.9 \pm 4.6$ & 0.00 \\
\hline \multicolumn{4}{|l|}{ Severity of pain } \\
\hline At the baseline & $3.3 \pm 0.9$ & $3.1 \pm 0.6$ & 0.19 \\
\hline After 4 weeks & $1.1 \pm 1.3$ & $2.0 \pm 1.0$ & 0.00 \\
\hline \multicolumn{4}{|l|}{ Pattern of pain, colic:continiuse } \\
\hline At baseline & $62: 3$ & $58: 2$ & 1.00 \\
\hline After 4 weeks & $31 / 2$ & $50 / 2$ & $<0.001$ \\
\hline \multicolumn{4}{|l|}{ Duration of pain (min/day) } \\
\hline At baseline & $22.5 \pm 50.8$ & $20.5 \pm 52.6$ & 0.25 \\
\hline After 4 weeks & $5.6 \pm 15.2$ & $15.4 \pm 42.4$ & $<0.001$ \\
\hline \multicolumn{4}{|l|}{ Dysfunction, presence:absence } \\
\hline At baseline & $13: 52$ & $8: 52$ & 0.34 \\
\hline After 4 weeks & $1 / 64$ & $6 / 54$ & 0.054 (fisher) \\
\hline \multicolumn{4}{|l|}{ Dysfunction (day/wk) } \\
\hline At baseline & $0.5 \pm 1.2$ & $0.2 \pm 0.7$ & 0.25 \\
\hline After 4 weeks & $0.05 \pm 0.3$ & $0.2 \pm 0.6$ & 0.04 \\
\hline \multicolumn{4}{|c|}{ Use another drug, used:not used } \\
\hline At baseline & $2 / 63$ & $0 / 60$ & 0.49 \\
\hline After 4 weeks & $1 / 64$ & $0 / 60$ & 1.00 (Fisher) \\
\hline \multicolumn{4}{|l|}{ Use another drug (day/wk) } \\
\hline At baseline & $0.1 \pm 0.6$ & 0 & 0.17 \\
\hline After 4 weeks & $0.03 \pm 0.2$ & 0 & 0.3 \\
\hline \multicolumn{4}{|l|}{ Initial diagnosis } \\
\hline Functional abdominal pain & $28(43)$ & $29(48)$ & 0.59 \\
\hline Functional dyspepsia & $16(24)$ & $13(21)$ & 0.83 \\
\hline Irritable bowel syndrome & $15(23)$ & $15(25)$ & 0.83 \\
\hline Abdominal migraine & $6(9)$ & $3(5)$ & 0.49 \\
\hline Treatment success & $32(49)$ & $8(13)$ & $<0.001$ \\
\hline
\end{tabular}

Values are presented as mean \pm standard deviation or number (\%).

$P$ values were derived using the Mann-Whitney, Wilcoxon, chi-square, or Fisher exact test.

Boldface indicates a statistically significant difference with $P<0.05$. 


\section{Functional abdominal pain}

Of 57 children presented with FAP, 28 children received probiotics, and 29 received placebo. Overall, treatment was successful in 13 patients in probiotic group and 8 patients in placebo group $(P=0.17)$. Frequency and duration of pain were not significantly reduced in probiotic group after 4 weeks of the treatment $(P=0.051$ and $P=0.054$, respectively). Severity of pain was significantly different in the 2 groups $(P<0.001)$ (Table 2$)$.

\section{Functional dyspepsia}

Of 29 patients presented with FD, 16 children received probiotic and 13 received placebo. Overall, 11 children (68\%) showed a significant response to probiotic treatment, compared to the placebo group $(P<0.001)$. Duration of pain, severity, and frequency were significantly reduced in the treatment group $(P<0.001, P<0.001$, and $P<0.001$, respectively) (Table 3$)$.

\section{Irritable bowel syndrome}

Of 30 children presented with IBS, 15 were in probiotic group and 15 were in placebo group. Six patients were successfully treated in the treatment group, while none of the patients in placebo group showed improvement $(P=0.01)$. Frequency, se-

Table 2. Characteristics of patients with the initial diagnosis of functional abdominal pain by study group

\begin{tabular}{|c|c|c|c|}
\hline Characteristic & $\begin{array}{l}\text { Case } \\
\text { group }\end{array}$ & $\begin{array}{l}\text { Control } \\
\text { group }\end{array}$ & $P$ value \\
\hline Treatment success & $13(46)$ & $8(27)$ & 0.17 \\
\hline \multicolumn{4}{|l|}{ Frequency of pain } \\
\hline At the baseline & $10.4 \pm 7.0$ & $7.4 \pm 5.1$ & 0.13 \\
\hline After 4 weeks & $2.1 \pm 2.7$ & $4.1 \pm 4.4$ & 0.051 \\
\hline \multicolumn{4}{|l|}{ Severity of pain } \\
\hline At the baseline & $3.5 \pm 1.0$ & $3.1 \pm 0.5$ & 0.14 \\
\hline After 4 weeks & $1.1 \pm 1.3$ & $2.0 \pm 1.0$ & $<0.001$ \\
\hline \multicolumn{4}{|c|}{ Pattern of pain, colic:continiuse } \\
\hline At baseline & $26: 2$ & $28: 1$ & 0.61 \\
\hline After 4 weeks & $13: 2$ & $20: 1$ & 0.22 \\
\hline \multicolumn{4}{|c|}{ Duration of pain (min/day) } \\
\hline At baseline & $36 \pm 75.6$ & $21.3 \pm 54$ & 0.16 \\
\hline After 4 weeks & $5.6 \pm 15.2$ & $15.4 \pm 42.4$ & 0.054 \\
\hline \multicolumn{4}{|c|}{ Dysfunction, presence:absence } \\
\hline At baseline & $7: 21$ & $3: 26$ & 0.17 \\
\hline After 4 weeks & $0: 28$ & $1: 28$ & 1.00 (Fischer) \\
\hline \multicolumn{4}{|l|}{ Dysfunction (day/wk) } \\
\hline At baseline & $0.6 \pm 1.4$ & $0.2 \pm 0.6$ & 0.14 \\
\hline After 4 weeks & 0 & $0.07 \pm 0.3$ & 0.32 \\
\hline \multicolumn{4}{|c|}{ Use another drug, used:not used } \\
\hline At baseline & $1: 27$ & $0: 29$ & 0.49 \\
\hline After 4 weeks & $0: 28$ & $0: 29$ & Invalid \\
\hline \multicolumn{4}{|c|}{ Use another drug (day/wk) } \\
\hline At baseline & $0.1 \pm 0.9$ & 0 & 0.31 \\
\hline After 4 weeks & 0 & 0 & Invalid \\
\hline
\end{tabular}

Values are presented as number (\%) or mean \pm standard deviation.

$P$ values were derived using the Mann-Whitney, Wilcoxon, chi-square, or Fisher exact test.

Boldface indicates a statistically significant difference with $P<0.05$. verity, and duration of pain were significantly reduced in treatment group, as compared to placebo $(P<0.001, P<0.001$, and $P<0.001$, respectively) (Table 4).

\section{Abdominal migraine}

Of 9 patients presented with AM, 6 children were in treatment group and 3 children were placebo. Overall, treatment was successful in 2 patients in probiotic group and none in placebo group, which was not statistically different $(P=0.5)$. Similarly, after 4 weeks, frequency, duration, and severity of pain were also not statistically significant in the 2 groups $(P>0.05)$ (Table 5$)$.

\section{Discussion}

Findings from this study reveal that probiotic treatment ( $L$. reuteri) significantly improved the intensity and the duration of RAP. All pain-related characteristics, such as the frequency of days of pain, the severity of pain, the duration of pain, and its pattern in the case group, were significantly reduced upon the treatment in comparison with the control group following the 4 weeks of the treatment. Also, the number of days of disturbance in daily activities, such as the absence of children from the school,

Table 3. Characteristics of patients with the initial diagnosis of functional dyspepsia by study group

\begin{tabular}{|c|c|c|c|}
\hline Characteristic & Probiotic & Placebo & $P$ value \\
\hline Treatment success & $11(68)$ & $0(0)$ & $<0.001$ \\
\hline \multicolumn{4}{|l|}{ Frequency of pain } \\
\hline At the baseline & $6.7 \pm 4.7$ & $9.7 \pm 7.3$ & 0.28 \\
\hline After 4 weeks & $1.6 \pm 3.0$ & $6.0 \pm 5.0$ & 0.00 \\
\hline \multicolumn{4}{|l|}{ Severity of pain } \\
\hline At the baseline & $3 \pm 0.8$ & $3 \pm 0.4$ & 0.65 \\
\hline After 4 weeks & $0.8 \pm 1.5$ & $2.0 \pm 0.6$ & $<0.001$ \\
\hline \multicolumn{4}{|c|}{ Pattern of pain, colic:continiuse } \\
\hline At baseline & $15: 1$ & $12: 1$ & 1.00 \\
\hline After 4 weeks & $5: 0$ & $12: 1$ & $<0.001$ \\
\hline \multicolumn{4}{|c|}{ Duration of pain (min/day) } \\
\hline At baseline & $14.5 \pm 10.8$ & $32.8 \pm 80.3$ & 0.94 \\
\hline After 4 weeks & $2.3 \pm 4.4$ & $24.9 \pm 64.7$ & $<0.001$ \\
\hline \multicolumn{4}{|c|}{ Dysfunction, presence:absence } \\
\hline At baseline & $1: 15$ & $1: 12$ & 1.00 \\
\hline After 4 weeks & $0: 16$ & $1: 12$ & 0.44 \\
\hline Dysfunction (day/wk) & 0.1 & & \\
\hline At baseline & $0.2 \pm 0.5$ & $0.08 \pm 0.2$ & 1.00 \\
\hline After 4 weeks & 0 & $0.08 \pm 0.2$ & 0.74 \\
\hline \multicolumn{4}{|c|}{ Use another drug, used:not used } \\
\hline At baseline & $1: 15$ & $0: 13$ & Invalid \\
\hline After 4 weeks & 0 & 0 & Invalid \\
\hline \multicolumn{4}{|c|}{ Use another drug (day/wk) } \\
\hline At baseline & $0.1 \pm 0.5$ & 0 & 0.77 \\
\hline After 4 weeks & 0 & 0 & Invalid \\
\hline
\end{tabular}

Values are presented as number (\%) or mean \pm standard deviation.

$P$ values were derived using the Mann-Whitney, Wilcoxon, chi-square, or Fisher exact test.

Boldface indicates a statistically significant difference with $P<0.05$. 
Table 4. Characteristics of patients with the initial diagnosis of irritable bowel syndrome by study group

\begin{tabular}{|c|c|c|c|}
\hline Characteristic & Probiotic & Placebo & $P$ value \\
\hline Treatment success & $6(40)$ & $0(0)$ & 0.01 \\
\hline \multicolumn{4}{|l|}{ Frequency of pain } \\
\hline At the baseline & $9.0 \pm 5.3$ & $8.3 \pm 6.1$ & 0.77 \\
\hline After 4 weeks & $3.7 \pm 5.5$ & $6.3 \pm 4.5$ & 0.01 \\
\hline \multicolumn{4}{|l|}{ Severity of pain } \\
\hline At the baseline & $3.4 \pm 0.8$ & $3.2 \pm 0.8$ & 0.46 \\
\hline After 4 weeks & $1.4 \pm 1.4$ & $2.8 \pm 0.8$ & 0.01 \\
\hline \multicolumn{4}{|c|}{ Pattern of pain, colic:continiuse } \\
\hline At baseline & $15: 0$ & $15: 0$ & Invalid \\
\hline After 4 weeks & $9: 6$ & $15: 0$ & 0.01 \\
\hline \multicolumn{4}{|c|}{ Duration of pain (min/day) } \\
\hline At baseline & $10.2 \pm 4.6$ & $10.6 \pm 4.9$ & 0.87 \\
\hline After 4 weeks & $2.9 \pm 4.0$ & $10.6 \pm 4.9$ & $<0.001$ \\
\hline \multicolumn{4}{|c|}{ Dysfunction, presence:absence } \\
\hline At baseline & $3: 12$ & $4: 11$ & 1.00 \\
\hline After 4 weeks & $1: 14$ & $4: 11$ & 0.33 \\
\hline Dysfunction (day/wk) & 0.6 & & \\
\hline At baseline & $0.7 \pm 1.3$ & $0.6 \pm 1.1$ & 0.87 \\
\hline After 4 weeks & $0.2 \pm 0.7$ & $0.6 \pm 1.1$ & 0.38 \\
\hline \multicolumn{4}{|c|}{ Use another drug, used:not used } \\
\hline At baseline & 0 & 0 & Invalid \\
\hline After 4 weeks & 0 & 0 & Invalid \\
\hline \multicolumn{4}{|c|}{ Use another drug (day/wk) } \\
\hline At baseline & 0 & 0 & Invalid \\
\hline After 4 weeks & 0 & 0 & Invalid \\
\hline
\end{tabular}

Values are presented as number (\%) or mean \pm standard deviation.

$P$ values were derived using the Mann-Whitney, Wilcoxon, chi-square, or Fisher exact test.

Boldface indicates a statistically significant difference with $P<0.05$.

was also reduced in the treatment group.

A recent study conducted by Royan et al. ${ }^{10)}$ in Iran reported that different strains of $L$. reuteri extracted from poultry ducks have immune-protective role in boiler chicken and balance serum lipid levels. Furthermore, Liu et al. ${ }^{11)}$ reported that $L$. renteri strains DSM 17938 and ATCC PTA 4659 have therapeutic efficacy against necrotizing enterocolitis as they promote antiinflammatory response by upregulating the production of IL-10 and reducing the expression of inflammatory cytokines such as; IL-6, tumor necrosis factor-alpha, toll-like receptor 4 , and nuclear factor kappa-light-chain-enhancer of activated B cell.

A recent Cochrane review update has shown low-to-moderate evidences based on the findings that probiotic treatment of RAP in children is associated with a significant reduction in the frequency and the intensity of the pain. This improvement was reported greatest in the children presented with IBS. Furthermore, Martens et al. ${ }^{12)}$ reported that Symbioflor2 containing $E$. coli significantly improves the symptoms of IBS among children aged $4-18$ years.

In a meta-analysis, Lactobacillus rhamnosus GG (LGG) have been reported to reduce the intensity of the pain in children presenting pain-associated functional gastrointestinal diseases. ${ }^{13)}$ Three clinical trials with a total population of 290 patients
Table 5. Characteristics of patients with the initial diagnosis of abdominal migraine by study group

\begin{tabular}{|c|c|c|c|}
\hline Characteristic & Case group & Control group & $P$ value \\
\hline Treatment success & $2(50)$ & $0(0)$ & 0.5 \\
\hline \multicolumn{4}{|l|}{ Frequency of pain } \\
\hline At the baseline & $4 \pm 2$ & $1.3 \pm 0.5$ & 0.02 \\
\hline After 4 weeks & $1.1 \pm 0.9$ & $1.3 \pm 0.5$ & 1.00 \\
\hline \multicolumn{4}{|l|}{ Severity of pain } \\
\hline At the baseline & $3.1 \pm 0.9$ & $2.6 \pm 0.5$ & 0.54 \\
\hline After 4 weeks & $1.3 \pm 1.5$ & $2.3 \pm 0.5$ & 0.26 \\
\hline \multicolumn{4}{|c|}{ Pattern of pain, colic:continiuse } \\
\hline At baseline & $6: 0$ & $3: 0$ & invalid \\
\hline After 4 weeks & 4:0 & $3: 0$ & 0.5 \\
\hline \multicolumn{4}{|c|}{ Duration of pain (min/day) } \\
\hline At baseline & $11.4 \pm 6.0$ & $8.3 \pm 2.6$ & 0.26 \\
\hline After 4 weeks & $11 \pm 24$ & $8.3 \pm 2.8$ & 0.16 \\
\hline \multicolumn{4}{|c|}{ Dysfunction, presence:absence } \\
\hline At baseline & $2: 4$ & $0: 3$ & 0.4 \\
\hline After 4 weeks & $0: 6$ & $0: 3$ & invalid \\
\hline \multicolumn{4}{|l|}{ Dysfunction (day/wk) } \\
\hline At baseline & $0.8 \pm 1.3$ & 0 & 0.54 \\
\hline After 4 weeks & 0 & 0 & 1.00 \\
\hline \multicolumn{4}{|c|}{ Use another drug, used:not used } \\
\hline At baseline & $0: 6$ & $0: 3$ & invalid \\
\hline After 4 weeks & $1: 5$ & $0: 3$ & 0.66 \\
\hline \multicolumn{4}{|c|}{ Use another drug (day/wk) } \\
\hline At baseline & 0 & 0 & Invalid \\
\hline After 4 weeks & $0.3 \pm 0.8$ & 0 & 0.74 \\
\hline
\end{tabular}

Values are presented as number (\%) or mean \pm standard deviation.

$P$ values were derived using the Mann-Whitney, Wilcoxon, chi-square, or Fisher exact test.

Boldface indicates a statistically significant difference with $P<0.05$.

were evaluated in the study. The results of this study showed success in treatment and a moderate reduction in the severity and frequency of the pain and the frequency of pain days in IBS patients. Since there are no established reports on the use of certain drugs in RAP pain management, this study reported different criteria for the treatment of RAP. ${ }^{14)}$

Our study results were similar to those of Gawrońska et al. ${ }^{15)}$ concerning the variables studied, the type of evaluation of pain-related functional disorders and the definition of pain severity; nevertheless, in this study, there was a relative success in probiotic-based treatment without significant improvement in recovery. Differences between the strains of bacteria used is a possible explanation of the discrepancies.

Romano et al. ${ }^{16)}$ L. reuteri DSM 17938 probiotic reduce the intensity of the pain among 6-16 years old children presented with functional abdominal pain.

L. reuteri is a lactic acid bacteria that has been shown to promote health by improving the movement, function, and modulation of pain signals via the neurotransmitter of the middle cerebral cortex. ${ }^{17,18)}$ It has been shown that $L$. reuteri has a high tendency to colonize in gastrointestinal mucus. ${ }^{19)}$

A clinical trial study in 2010 by Savino et al. ${ }^{20)}$ was conducted to evaluate the effect of $L$. reuteri DSM 17938 for the treatment 
of neonatal colic. Our study is the first clinical trial to report highdose $L$. reuteri treatment for abdominal incontinence in children. This result was obtained not only in our study population with abdominal remission but also in each subgroup including FAP, FD, and IBS. In abdominal migraine, the difference between the studied variables in the case and control groups was not significant, as one of the causes was the low number of patients in this subgroup ( 9 subjects).

In conclusion, owing to the facts that RAP is one of the common disorders in children, especially at school-going age, and can disrupt daily activities, leading to fewer children attending in the community activities, and also adding to anxiety for the parents along with socioeconomic pressure, therapeutic measures to for RAP stand great demand. Based on the results of this study, $L$. reuteri probiotic treatment significantly improves the incidence of abdominal pain in children. Considering the results of this study and its significant effects, especially on IBS and FD, the use of probiotic $L$. reuteri can be recommended as one of the RAP therapies.

Given that there is no known mechanism of action for L. reuteri that makes $L$. reuteri superior to LGG and other probiotics, detailed and comparative case-control trials are recommended to draw a clinically advantageous conclusion.

Further evidence-based studies on the therapeutic use of probiotics in patients with a larger sample size along with their dosage and the duration of the treatment are also recommended.

\section{Conflicts of interest}

No potential conflict of interest relevant to this article was reported.

\section{Acknowledgments}

We greatly thank "Tehran University of Medical Sciences, Tehran, Iran" for their cooperation and support.

\section{References}

1. Rahmani P, Moradzadeh A, Farahmand F. Giving probiotics to your children for gastrointestinal problems: In the light of scientific findings. PharmaNutrition 2019;10:100164.

2. de Moreno de LeBlanc A, Del Carmen S, Chatel JM, Miyoshi A, Azevedo V, Langella P, et al. Current review of genetically modified lactic acid bacteria for the prevention and treatment of colitis using murine models. Gastroenterol Res Pract 2015;2015:146972.

3. Peeters B. Gastrointestinal motility disorders in children: etiology and associated behaviors [dissertation]. Amsterdam: University of Amsterdam, 2013.
4. Salem AE, Singh R, Ayoub YK, Khairy AM, Mullin GE. The gut microbiome and irritable bowel syndrome: State of art review. Arab J Gastroenterol 2018;19:136-41.

5. Marcdante K, Kliegman RM. Nelson essentials of pediatrics e-book: with student consult online access: Philadelphia (PA): Saunders/Elsevier, 2014.

6. Newlove-Delgado TV, Martin AE, Abbott RA, Bethel A, ThompsonCoon J, Whear R, et al. Dietary interventions for recurrent abdominal pain in childhood. Cochrane Database Syst Rev 2017;3:CD010972.

7. Bufler P, Gross M, Uhlig HH. Recurrent abdominal pain in childhood. Dtsch Arztebl Int 2011;108:295-304.

8. Rose MA. Lactobacillus rhamnosus GG reduces frequency and severity of abdominal pain compared with placebo in children with irritable bowel syndrome. Evid Based Med 2011;16:141-2.

9. Shih DQ, Kwan LY. All roads lead to Rome: update on Rome III criteria and new treatment options. Gastroenterol Rep 2007;1:56-65.

10. Royan M, Hashemi M, Seighalani R. Effect of isolates of lactobacillus reuteri and lactobacillus salivarius isolated from the gastrointestinal tract of native poultry of northern of iran on performance, serum lipids and immune parameters of broiler chickens. Res Anim Prod 2019;10:18-26

11. Liu Y, Fatheree NY, Mangalat N, Rhoads JM. Lactobacillus reuteri strains reduce incidence and severity of experimental necrotizing enterocolitis via modulation of TLR4 and NF- $\mathrm{KB}$ signaling in the intestine. Am J Physiol Gastrointest Liver Physiol 2012;302:G608-17.

12. Martens U, Enck P, Zieseniss E. Probiotic treatment of irritable bowel syndrome in children. Ger Med Sci 2010;8:Doc07.

13. Horvath A, Dziechciarz P, Szajewska H. Meta-analysis: Lactobacillus rhamnosus GG for abdominal pain-related functional gastrointestinal disorders in childhood. Aliment Pharmacol Ther 2011;33:1302-10.

14. Francavilla R, Miniello V, Magistà AM, De Canio A, Bucci N, Gagliardi F, et al. A randomized controlled trial of Lactobacillus GG in children with functional abdominal pain. Pediatrics 2010;126:e1445-52.

15. Gawrońska A, Dziechciarz P, Horvath A, Szajewska H. A randomized double-blind placebo-controlled trial of Lactobacillus GG for abdominal pain disorders in children. Aliment Pharmacol Ther 2007;25:177-84.

16. Romano C, Ferrau' V, Cavataio F, Iacono G, Spina M, Lionetti E, et al. Lactobacillus reuteri in children with functional abdominal pain (FAP). J Paediatr Child Health 2014;50:E68-71.

17. Wang B, Mao YK, Diorio C, Wang L, Huizinga JD, Bienenstock J, et al. Lactobacillus reuteri ingestion and IK(Ca) channel blockade have similar effects on rat colon motility and myenteric neurones. Neurogastroenterol Motil 2010;22:98-107, e33.

18. Indrio F, Riezzo G, Raimondi F, Bisceglia M, Cavallo L, Francavilla R. The effects of probiotics on feeding tolerance, bowel habits, and gastrointestinal motility in preterm newborns. J Pediatr 2008;152:801-6.

19. Valeur N, Engel P, Carbajal N, Connolly E, Ladefoged K. Colonization and immunomodulation by Lactobacillus reuteri ATCC 55730 in the human gastrointestinal tract. Appl Environ Microbiol 2004;70:1176-81.

20. Savino F, Cordisco L, Tarasco V, Palumeri E, Calabrese R, Oggero R, et al. Lactobacillus reuteri DSM 17938 in infantile colic: a randomized, doubleblind, placebo-controlled trial. Pediatrics 2010;126:e526-33.

How to cite this article: Rahmani P, Ghouran-orimi A, Motamed F, Moradzadeh A. Evaluating the effects of probiotics in pediatrics with recurrent abdominal pain. Clin Exp Pediatr 2020;63:485-90. https://doi.org/10.3345/cep2019.01613 